\title{
烷基磺酸酯基功能化离子液体的合成、性质及其 在酯化反应中的应用
}

\author{
赵应伟 ${ }^{1,2}$, 李 臻 $^{1}$, 夏春谷 ${ }^{1}$ \\ 1 中国科学院兰州化学物理研究所崖基合成与选择氧化国家重点实验室, 甘肃兰州 730000 \\ 2 中国科学院研究生院, 北京 100049
}

摘要：采用廉价易得的原料合成了阳离子含有烷基磺酸酯官能团的功能化离子液体,并考察了其热稳定性. 该离子液体可催化 羧酸的酯化反应, 其催化活性高于阳离子非功能化的离子液体, 而与磺酸功能化离子液体相当. 同时采用电喷雾电离质谱考察 了该离子液体在酯化反应中的作用.

关键词: 离子液体; 烷基磺酸酯; 酯化

中图分类号：0643 文献标识码：A

\section{Alkyl Sulfonate Functionalized Ionic Liquids: Synthesis, Properties, and Their Application in Esterification}

\author{
ZHAO Yingwei ${ }^{1,2}$, LI Zhen ${ }^{1, *}$, XIA Chungu ${ }^{1, *}$ \\ ${ }^{1}$ State Key Laboratory for Oxo Synthesis and Selective Oxidation, Lanzhou Institute of Chemical Physics, \\ Chinese Academy of Sciences, Lanzhou 730000, Gansu, China \\ ${ }^{2}$ Graduate University of Chinese Academy of Sciences, Beijing 100049, China
}

\begin{abstract}
Ionic liquids based on alkyl sulfate anions and alkyl sulfonate functionalized cations were prepared from commercially available and cheap materials and their thermal-instability properties were studied in detail. These ionic liquids were active catalysts for esterification and they gave moderate to high yields. The catalytic performance was found to be much better than that of conventional non-cation functionalized ionic liquids and close to sulfonic acid functionalized ionic liquids. The catalytic process in this reaction was investigated by electrospray ionization mass spectrometry and a plausible reaction mechanism is suggested.
\end{abstract}

Key words: ionic liquid; alkylsulfonate; esterification

Room temperature ionic liquids (ILs) have been extensively studied as solvents and catalysts in organic chemistry [1-3]. Diversified ionic liquids containing various cations and anions have been synthesized over the past few decades. Recently, an increasing amount of attempts have been made to develop functionalized ionic liquids (FILs) by the incorporation of functional groups as a part of the cation and/or anion [4]. In 2002, a series of low cost and halide-free ionic liquids containing alkyl sulfate anions were prepared by Holbrey et al. [5]. They were later used as solvents in many reactions [6-9] and their physical properties were extensively studied [10-12]. However, they are not regarded as FILs because of the catalytic inertia of the sulfate anions.

Our efforts have focused on the further functionalization of this type of ionic liquid by the alkylation of zwitterions (obtained by the reaction of 1-methylimdazole and 1,4-butane sultone [13]) using dimethyl sulfate or diethyl sulfate. We have previously produced the new cation-functionalized ionic liquids 1-(4-methyl sulfonate)-butyl-3- methylimidazolium methyl sulfate (1) and 1-(4-ethyl sulfonate)-butyl-3-ethylimidazolium ethyl sulfate (2) [14] Herein, we report detailed studies on the synthesis and properties of these alkyl sulfonate functionalized ionic liq-

Received date 29 September 2010. Accepted 13 December 2010

*Corresponding author. Tel/Fax: +86-931-4968129; E-mail: cgxia@licp.cas.cn, zhenli@licp.cas.cn

Foundation item: Supported by the National Natural Science Fund for Distinguished Young Scholars (20625308).

English edition available online at ScienceDirect (http://www.sciencedirect.com/science/journal/18722067). 
uids. Esterification is one of the most important reactions in organic synthesis for the preparation of fine chemicals and people have carried out reactions using various categories of ionic liquid solvents [15-17]. The disadvantage of this strategy is the high consumption of ionic liquids. For the functionalized cations, relatively high catalytic efficiencies were expected for ionic liquids $\mathbf{1}$ and $\mathbf{2}$. In this paper, the application of these ionic liquids as catalysts in esterification was also studied. For comparison, some known ionic liquids with similar structures to ionic liquids 1 and 2 (3-7) were also prepared and used in this reaction (Scheme 1).

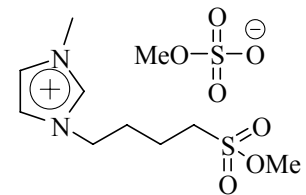

1

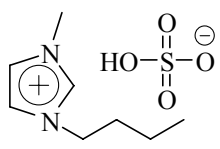

5

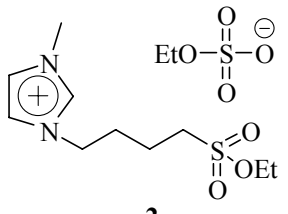

2

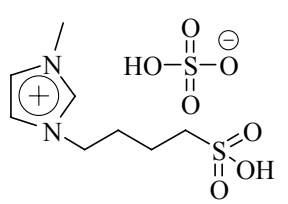

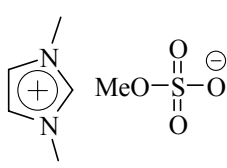

3

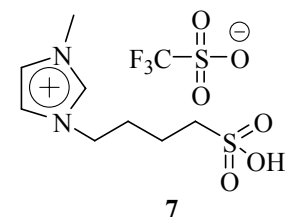

Scheme 1. The ionic liquids discussed in this article.

\section{Experimental}

\subsection{Synthesis of ionic liquids}

Dimethyl sulfate, diethyl sulfate, and 1-methylimidazole were redistilled under reduced pressure before use. 1,4-Butane sultone and the other reagents were used as received without further purification. The ionic liquids $\mathbf{1}$ and 2 were prepared using 1-methylimidazole and dimethyl sulfate or diethyl sulfate under solvent-free conditions [14] (Scheme 2). The preparation and purification of ionic liquids 3-7 and methanesulfonate methyl ester were achieved according to the reported methods [5,18-21].

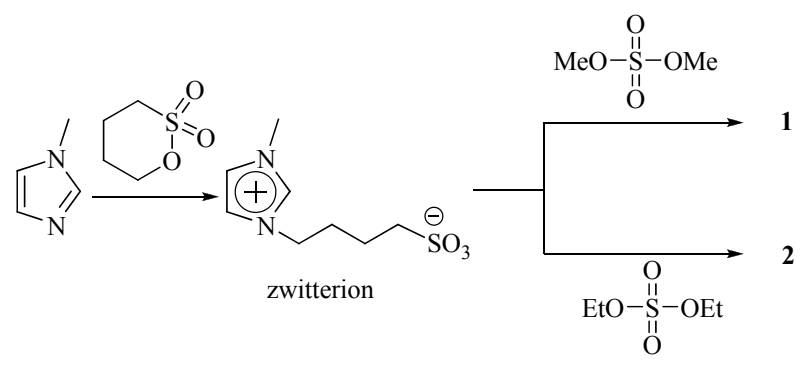

Scheme 2. Synthesis of ionic liquids $\mathbf{1}$ and 2.

\subsection{Characterization of ionic liquids}

NMR spectra were recorded on an INOVA-400 spectrometer. Thermal decomposition profiles were collected by thermogravimetric analysis (TGA, Diamond TG/DTA/DSC, $10{ }^{\circ} \mathrm{C} / \mathrm{min}$ heating rate under nitrogen). The $\mathrm{pH}$ values were recorded using a DELTA $320 \mathrm{pH}$ meter. Electrospray ionization mass spectrometry (ESI-MS) analysis was performed using a Waters micromass ZQ alliance spectrometer (capillary $3.6 \mathrm{kV}$, cone $10 \mathrm{~V}$, source temperature $100{ }^{\circ} \mathrm{C}$, desolvation temperature $120^{\circ} \mathrm{C}$, data format $\mathrm{MCA}$, flow velocity $10 \mu \mathrm{l} / \mathrm{min})$.

\subsection{Typical esterification procedure}

Carboxylic acid and methanol were added to the ionic liquid and then the mixture was heated with stirring at 80 ${ }^{\circ} \mathrm{C}$. For the low boiling point esters, after the reaction the distillation equipment was set up and the desired ester as well as unreacted methanol was distilled at $100{ }^{\circ} \mathrm{C}$. The mixture obtained from the distillation was dried over anhydrous $\mathrm{Na}_{2} \mathrm{SO}_{4}$. The yield was obtained from an Agilent 6820 $\mathrm{GC}$ according to the ratio of the chromatographic peak area of the product and an internal standard. For the high boiling point esters, water and unreacted methanol were evaporated under vacuum. The ether was then added and the ionic liquid was isolated. The ether layer was washed with saturated aqueous $\mathrm{NaHCO}_{3}$ and dried over anhydrous $\mathrm{Na}_{2} \mathrm{SO}_{4}$. Finally, the ether was removed at $30{ }^{\circ} \mathrm{C}$ under vacuum and the yield of ester was calculated. For the synthesis of dimethyl fumarate, the white solid product was isolated and washed with cold saturated aqueous $\mathrm{NaHCO}_{3}$ after the reaction. Finally it was dried at $70{ }^{\circ} \mathrm{C}$ for $4 \mathrm{~h}$ in an oven. All the products were qualitatively determined by an HP 6890/5973 GC-MS.

\subsection{Reactions of ionic liquids with phenylacetic acid}

The ionic liquid $(5 \mathrm{mmol})$ and phenylacetic acid (15 mmol) were charged into a $25 \mathrm{ml}$ round bottom flask and 
heated with stirring at $80{ }^{\circ} \mathrm{C}$ for $4 \mathrm{~h}$. Cold anhydrous ether was then added. The obtained viscous liquid was washed with anhydrous ether $(15 \mathrm{ml} \times 5)$ to remove the phenylacetic acid methyl ester formed in the reaction and to remove unreacted phenylacetic acid. The ether was then distilled under vacuum for $4 \mathrm{~h}$. Finally the obtained sample was dissolved in acetonitrile before analysis by ESI-MS.

\subsection{Determination of the Hammett acidity function of the ionic liquids}

The ionic liquids and the indicator 4-nitroaniline were dissolved in dry $\mathrm{CH}_{2} \mathrm{Cl}_{2}$ at concentrations of $5 \mathrm{mmol} / \mathrm{L}$ and $0.15 \mathrm{mmol} / \mathrm{L}$, respectively. When dimethyl yellow was used as an indicator its concentration was $0.085 \mathrm{mmol} / \mathrm{L}$. The solutions were shaken vigorously and then left to stand for 6 h. Their UV-Vis spectra were recorded on an HP 8453 UV-Vis spectrophotometer.

\section{Results and discussion}

\subsection{Synthesis of ionic liquids}

For the preparation of these alkyl sulfonate functionalized ionic liquids, it should be noted that commercially available dimethyl sulfate and diethyl sulfate must be redistilled before use because trace sulfuric acid will lead to the formation of 1-(4-sulfonic acid)-butyl-3-methylimidazolium hydrogen sulfate (7) [20] in the reaction. We found that heating is required to promote the reaction of the zwitterion with dimethyl sulfate or diethyl sulfate indicating that the zwitterion is a relatively weak nucleophile and fairly inert to electrophilic reagents. The synthesis of the alkylimidazolium methyl sulfates $\mathbf{3}$ and $\mathbf{4}$ requires no heat but does require an ice-bath as the preparation reactions are highly exothermic [5]. We also previously found that relatively weak alkylating agents such as iodomethane did not react with the zwitterion while alkylimidazole was easily alkylated [22]. On the other hand, the zwitterion obtained from the reactions of pyridine, tributylphosphine, or triethylamine with 1,4-butane sultone could react with dimethyl sulfate or diethyl sulfate under heating resulting in the formation of the corresponding sulfonate functionalized ionic liquids.

\subsection{TGA results}

The thermal instability of the alkylimidazolium methyl sulfate ionic liquid 3 has been recognized previously [5]. To characterize the thermal degradation of the sulfonate functionalized ionic liquids $\mathbf{1}$ and 2, TGA were performed and the results are shown in Fig. 1. For comparison, the TGA trace of the zwitterion was also investigated. A multi-step decomposition process was found for alkylimidazolium alkyl sulfate ionic liquids and this has been reported previously [5]. In our work, the two sulfonate-functionalized ionic liquids showed a similar thermal decomposition process consisting of gradual and continuous weight loss between 100 and $330{ }^{\circ} \mathrm{C}$. This was followed by a major weight loss event around $330-380{ }^{\circ} \mathrm{C}$. In the first step, ionic liquid $\mathbf{1}$ showed a mass loss above $30 \%$ and ionic liquid 2 lost about $40 \%$. These losses are in accordance with the weight proportion of $\left(\mathrm{CH}_{3}\right)_{2} \mathrm{SO}_{4}$ in ionic liquid $\mathbf{1}$ and $\left(\mathrm{C}_{2} \mathrm{H}_{5}\right)_{2} \mathrm{SO}_{4}$ in ionic liquid $2(36.6 \%$ and $41.4 \%$, respectively). The TGA curve of the zwitterion provides more evidence for the release of dimethyl sulfate or ethyl sulfate from the ionic liquids. Figure 1 shows that at temperatures above $330{ }^{\circ} \mathrm{C}$ the TGA curves of both ionic liquids $\mathbf{1}$ and 2 gradually converge with that of the zwitterion.

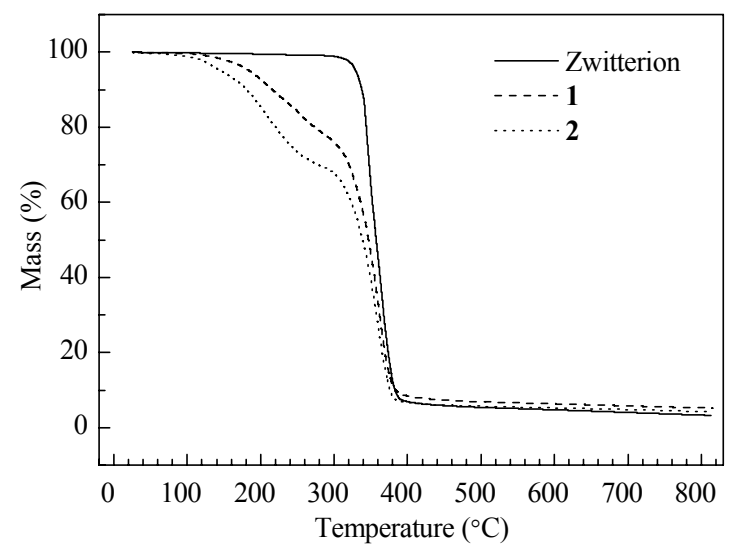

Fig. 1. TG curves for the two ionic liquids and the zwitterion.

\subsection{Catalytic activity during esterification}

The esterification of a carboxylic acid with an alcohol is typically catalyzed by a Brönsted acid or a Lewis acid. We have already shown that the catalytic performance of a Brönsted acidic ionic liquid is strongly dependent on its Brönsted acidity [23]. To our surprise, without Brönsted or Lewis acidic functional groups in their structures, ionic liquids $\mathbf{1}$ and $\mathbf{2}$ showed catalytic activity for the esterification of carboxylic acids.

The results listed in Table 1 suggest that for all the carboxylic acid substrates the catalytic performances of $\mathbf{1}$ and $\mathbf{2}$ are much better than that of the conventional non-cation functionalized ionic liquids $\mathbf{3}, \mathbf{4}$, and $\mathbf{5}$, and close to that of the sulfonic acid functionalized ionic liquids $\mathbf{6}$ and 7 and sulfuric acid at a low catalyst loading (Table 1, entries 1-16). For the esterification of benzoic acid, the yield of ester obtained by ionic liquid $\mathbf{1}$ is even better than that obtained by sulfuric acid (Table 1, entries 17 and 18). Moderate to high yields were still obtained when the molar ratio of methanol/carboxyl group was reduced to 1:1 (Table 1, en- 
Table 1 Esterification of a carboxylic acid with methanol in the presence of sulfonate functionalized ionic liquids and other related ionic liquids

\begin{tabular}{|c|c|c|c|c|c|c|}
\hline Entry & Carboxylic acid & Catalyst & Catalyst loading (mol\%) & Acid:alcohol & Time (h) & Yield (\%) \\
\hline 1 & $n$-butyric acid & 1 & 0.5 & $1: 4$ & 2 & 76 \\
\hline 2 & $n$-butyric acid & 3 & 0.5 & $1: 4$ & 2 & 4 \\
\hline 4 & $n$-butyric acid & 5 & 0.5 & $1: 4$ & 2 & 56 \\
\hline 5 & $n$-butyric acid & 6 & 0.5 & $1: 4$ & 2 & 92 \\
\hline 6 & $n$-butyric acid & 7 & 0.5 & $1: 4$ & 2 & 93 \\
\hline 7 & $n$-butyric acid & $\mathrm{H}_{2} \mathrm{SO}_{4}$ & 0.5 & $1: 4$ & 2 & 84 \\
\hline 8 & $n$-butyric acid & 1 & 1 & $1: 4$ & 2 & 93 \\
\hline 9 & n-butyric acid & 6 & 1 & $1: 4$ & 2 & 96 \\
\hline 10 & phenylacetic acid & 1 & 1 & $1: 4$ & 4 & $85 / 84^{\mathrm{a}}$ \\
\hline 11 & phenylacetic acid & 2 & 1 & $1: 4$ & 4 & 78 \\
\hline 12 & phenylacetic acid & 3 & 1 & $1: 4$ & 4 & 23 \\
\hline 14 & phenylacetic acid & 5 & 1 & $1: 4$ & 4 & 56 \\
\hline 15 & phenylacetic acid & 6 & 1 & $1: 4$ & 4 & 82 \\
\hline 16 & phenylacetic acid & $\mathrm{H}_{2} \mathrm{SO}_{4}$ & 1 & $1: 4$ & 4 & 85 \\
\hline 17 & benzoic acid & 1 & 1 & $1: 4$ & 4 & 68 \\
\hline 18 & benzoic acid & 6 & 1 & $1: 4$ & 4 & 53 \\
\hline 19 & benzoic acid & 1 & 10 & $1: 2$ & 4 & 86 \\
\hline 20 & $n$-butyric acid & 1 & 10 & $1: 1$ & 2 & $89 / 84^{b}$ \\
\hline 21 & phenylacetic acid & 1 & 10 & $1: 1$ & 4 & 86 \\
\hline $22^{\mathrm{c}}$ & acetic acid & 2 & 20 & $1: 1$ & 4 & 91 \\
\hline $23^{c}$ & acetic acid & 7 & 20 & $1: 1$ & 4 & 93 \\
\hline 24 & fumaric acid & 1 & 20 & $1: 2$ & 4 & $90^{\mathrm{d}}$ \\
\hline 25 & malonic acid & 1 & 20 & $1: 2$ & 4 & $64^{\mathrm{d}}$ \\
\hline
\end{tabular}

Reaction conditions: $80^{\circ} \mathrm{C}$. ${ }^{\text {a }}$ The fifth run $(2$ nd run $87 \%$, 3rd run $84 \%$, 4 th run $84 \%) .{ }^{b}$ The second run. ${ }^{c}$ Esterification with ethanol. ${ }^{\mathrm{d}}$ Yield of diester.

tries 20-26). In these cases, the increase of catalyst loading to 10 or $20 \mathrm{~mol} \%$ (relative to the carboxyl group) was needed to create a biphasic catalytic system, which promoted the equilibrium process in favor of the products side. The ethyl sulfonate functionalized ionic liquid $\mathbf{2}$ also exhibited comparable performance in the esterification of acetic acid and ethanol to that obtained with ionic liquid 7 (Table 1, entries 22 and 23).

As the ionic liquid and the ester were immiscible, the ionic liquid was easily separated by simple decantation after the excess alcohol was distilled off, it could be reused directly without any treatment. Almost no decrease in ester yield was obtained after the ionic liquid $\mathbf{1}$ was reused for four times (Table 1, entry 10).

To investigate the role of the sulfonate-functionalized ionic liquids in the esterification process, we first determined their Hammett acidity functions in $\mathrm{CH}_{2} \mathrm{Cl}_{2}$ using 4-nitroaniline $\left(\mathrm{p} K(\mathrm{I})_{\mathrm{aq}}=0.99\right)$ and dimethyl yellow $\left(\mathrm{p} K(\mathrm{I})_{\mathrm{aq}}\right.$ $=3.3$ ) as indicators. However, no absorbance decrease was found for the indicators. This clearly indicates that the sulfonate-functionalized ionic liquids $\mathbf{1}$ and $\mathbf{2}$ exhibit nearly no Brönsted acidity [23].

In further experiments we found that when a large amount of ethylsulfonate functionalized ionic liquid $\mathbf{2}$ was used in the methyl esterification of phenylacetic acid, an ethyl ester was present (Scheme 3, top). On the other hand, when the methylsulfonate-functionalized ionic liquid $\mathbf{1}$ was used in the ethyl esterification of pheylacetic acid, a methyl ester was formed (Scheme 3, bottom). This phenomenon led us to consider that the Brönsted acid center might be formed by the reaction of the sulfonate functionalized ionic liquid and the carboxylic acid substrate. We then mixed ionic liquid 1 with phenylacetic acid and heated without solvent. ESI-MS and H-NMR analysis of the ionic liquid phase and a GC-MS analysis of the organic phase indicated that the phenylacetic acid was methylated to afford the corresponding methyl ester. For ionic liquid 1, the methyl sulfate anion $\left(m / z=110.8, \delta\left(\mathrm{CH}_{3} \mathrm{SO}_{4}\right)=3.14\right)$ transformed to a hydrogen sulfate $(m / z=97.0)$ completely in $4 \mathrm{~h}$ at $80{ }^{\circ} \mathrm{C}$ but only a small amount of the methyl sulfonate group in the cation $\left(m / z=233.2, \delta\left(\mathrm{C}_{4} \mathrm{H}_{6} \mathrm{~N}_{2}\left(\mathrm{CH}_{2}\right)_{4} \mathrm{SO}_{3} \mathrm{CH}_{3}\right)=3.37\right)$ transformed to sulfonate acid group $(\mathrm{m} / z=219.2)$. This result led us to consider whether hydrogen sulfate was the real catalytic species. However, the catalytic efficiency of ionic liquid 5 with the anion of the hydrogen sulfate but without cation-functionalization during esterification was much 


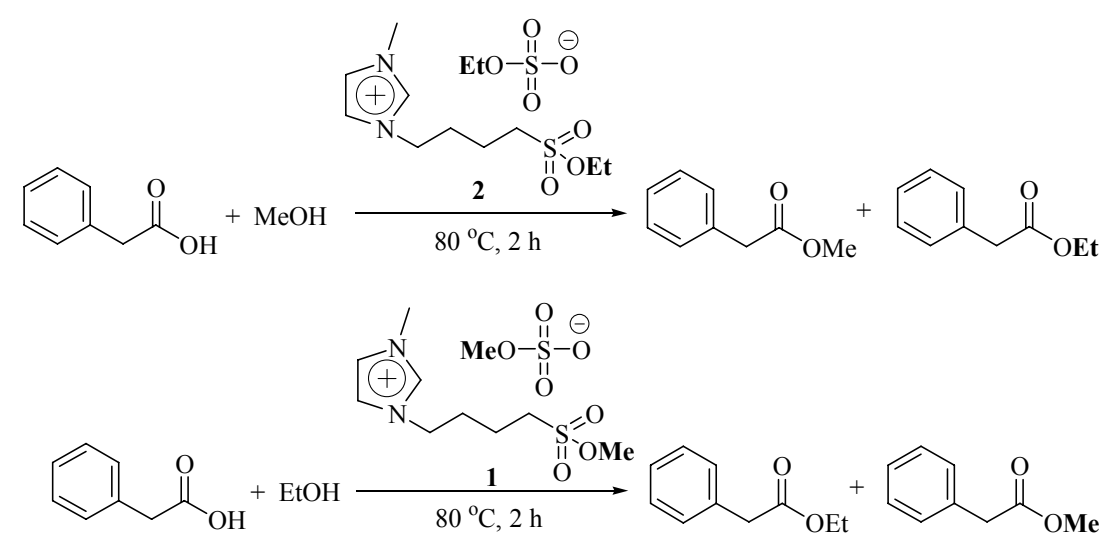

Scheme 3. Formation of by-products in the esterification catalyzed by the sulfonate-functionalized ionic liquids.

lower than that of ionic liquids $\mathbf{1}$ and $\mathbf{6}$ (Table 1, entries 4 and 14). Ionic liquids 3 and $\mathbf{4}$ with the methyl sulfate anion also exhibited low catalytic activity and these were even worse than that of ionic liquid 5 (Table 1, entries 2, 3, 12, and 13) although their anion could also be transformed into hydrogen sulfate in the presence of a carboxylic acid. This was shown by ESI-MS. A reasonable explanation is that the sulfonic acid group played a crucial role despite its low concentration.

However, another factor that should be considered is the water produced in the esterification system. The hydrolysis of alkylsulfate ionic liquids such as $\mathbf{3}$ and $\mathbf{4}$ leads to the formation of $\mathrm{HSO}_{4}^{-}$and therefore the $\mathrm{pH}$ of their aqueous solutions should decrease [24]. Therefore, the $\mathrm{pH}$ of these ionic liquids in water was measured and the results shown in Table 2. We found that ionic liquid $\mathbf{3}$, with a methyl sulfate anion and the methanesulfonate methyl ester, which has the same functional group as the cation of ionic liquid 1, were hydrolyzed to a low extent. However, ionic liquid 1, which contained a combined methyl sulfate anion and methyl sulfonate functionalized cation, was strongly acidic in water, which is similar to the acidic ionic liquids 5 and $\mathbf{6}$. The aqueous solution of ionic liquid $\mathbf{2}$ is also acidic (Table 2 , entry 2). The reason remains unclear but is probably re-

Table $2 \mathrm{pH}$ of the ionic liquids at room temperature

\begin{tabular}{ccc}
\hline Entry & Ionic liquid & $\mathrm{pH}^{\mathrm{a}}$ \\
\hline 1 & $\mathbf{1}$ & $2.72(2.51)^{\mathrm{b}}$ \\
2 & $\mathbf{2}$ & 3.14 \\
3 & $\mathbf{3}$ & 7.50 \\
4 & $\mathbf{5}$ & 2.11 \\
5 & $\mathbf{6}$ & 1.89 \\
6 & $\mathrm{CH}_{3} \mathrm{SO}_{3} \mathrm{CH}_{3}$ & 4.44 \\
\hline
\end{tabular}

${ }^{\mathrm{a}} \mathrm{pH}$ values were determined at $16{ }^{\circ} \mathrm{C}$ with $0.01 \mathrm{~mol} / \mathrm{L}$ ionic liquid in water. After the ionic liquids were dissolved in water the solutions were allowed to stand for $0.5 \mathrm{~h}$ and then the $\mathrm{pH}$ was determined.

${ }^{\mathrm{b}}$ The ionic liquid solution was allowed to stand for $20 \mathrm{~h}$ before $\mathrm{pH}$ determination. lated to the interaction between the cation and the anion. ESI-MS showed that the methyl sulfonate group in the cation is hydrolyzed to a sulfonic group to a high degree and the methyl sulfate anion simultaneously transforms to hydrogen sulfate.

Therefore, we can generally describe the reaction process as below: initially, the anion methyl sulfate of ionic liquid $\mathbf{1}$ reacts with the carboxylic acid to form hydrogen sulfate. Esterification was catalyzed by a relatively weak Brönsted acid hydrogen sulfate and water was formed leading to the hydrolysis of ionic liquid 1. Finally, the strong Brönsted acid center that formed (sulfonate acid group in the cation) remarkably accelerated the reaction rate. Strictly speaking, ionic liquid $\mathbf{1}$ should not be regarded as a real catalyst but a precursor of a catalyst as its structure changed after the reaction.

\section{Conclusions}

We describe the synthesis of a series of ionic liquids containing both an alkyl sulfate anion and an alkyl sulfonate functionalized cation. Because dimethyl sulfate and diethyl sulfate are strong alkylating reagents, the preparation of the sulfonate functionalized ionic liquids proceeds smoothly and with excellent yields. We found that these ionic liquids were thermally unstable and decomposed easily to zwitterions at high temperature and they were also easily hydrolyzed. Although these ionic liquids have no acidic group they were efficient catalysts for esterification. Control experiments and ESI-MS studies showed that the hydrolyzation of the methyl sulfonate group in the cation of ionic liquid $\mathbf{1}$ to form a strong Brönsted acid center played a crucial role in promoting the esterification process.

\section{References}

1 Welton T. Chem Rev, 1999, 99: 2071 
2 Chowdhury S, Mohan R S, Scott J L. Tetrahedron, 2007, 63: 2363

3 Parvulescu V I, Hardacre C. Chem Rev, 2007, 107: 2615

4 Lee S G. Chem Commun, 2006: 1049

5 Holbrey J D, Reichert W M, Swatloski R P, Broker G A, Pitner W R, Seddon K R, Rogers R D. Green Chem, 2002, 4: 407

6 Klingshirn M A, Rogers R D, Shaughnessy K H. J Organomet Chem, 2005, 690: 3620

7 Teijeira M, Fall Y, Santamarta F, Tojo E. Tetrahedron Lett, 2007, 48: 7926

8 Bosmann A, Datsevich L, Jess A, Lauter A, Schmitz C, Wasserscheid P. Chem Commun, 2001: 2494

9 Arce A, Rodriguez H, Soto A. Green Chem, 2007, 9: 247

10 Gomez E, Gonzalez B, Calvar N, Tojo E, Dominguez A. $J$ Chem Eng Data, 2006, 51: 2096

11 Pereiro A B, Verdia P, Tojo E, Rodriguez A. J Chem Eng Data, 2007, 52: 377

12 Pereiro A B, Santamarta F, Tojo E, Rodriguez A, Tojo J. $J$ Chem Eng Data, 2006, 51: 952

13 Cole A C, Jensen J L, Ntai I, Tran K L T, Weaver K J, Forbes
D C, Davis J H Jr. J Am Chem Soc, 2002, 124: 5962

14 Liu J, Li Z, Chen J, Xia C. Catal Commun, 2009, 10: 799

15 Fraga-Dubreuil J, Bourahla K, Rahmouni M, Bazureau J P, Hamelin J. Catal Commun, 2002, 3: 185

16 Joseph T, Sahoo S, Halligudi S B. J Mol Catal A, 2005, 234: 107

17 Fang D, Zhou X L, Ye Z W, Liu Z L. Ind Eng Chem Res, 2006, 45: 7982

18 Zhao G Y, Jiang T, Gao H X, Han B, Huang J, Sun D H. Green Chem, 2004, 6: 75

19 Gu Y, Shi F, Deng Y. Catal Commun, 2003, 4: 597

20 Gui J Z, Cong X H, Liu D, Zhang X T, Hu Z D, Sun Z L. Catal Commun, 2004, 5: 473

21 Smaldone R A, Moore J S. J Am Chem Soc, 2007, 129: 5444

22 Kondo Y, Izawa S, Kusabayashi S. J Chem Soc, Perkin Trans 2, 1988: 1925

23 Zhao Y, Long J, Deng F, Liu X, Li Z, Xia C, Peng J. Catal Commun, 2009, 10: 732

24 Himmler S, Hormann S, van Hal R, Schulz P S, Wasserscheid P. Green Chem, 2006, 8: 887 